\title{
Extrusion Granulation
}

National Cancer Institute

\section{Source}

National Cancer Institute. Extrusion Granulation. NCI Thesaurus. Code C113007.

The use of a series of integrated steps whereby a dry powder is conditioned to form a plasticized mass that is forced through a screen and cut into sections, ultimately to yield granules possessing characteristic dimensions and density. 